\title{
NUEVA ESPECIE DE MASTIGOPROCTUS POCOCK, 1894 (THELYPHONIDA, THELYPHONIDAE) DE VENEZUELA NOROCCIDENTAL
}

\author{
O. Villarreal Manzanilla ${ }^{1}$ \& A. P. de L. Giupponi²,3
}

\begin{abstract}
RESUMEN
Una nueva especie del género Mastigoproctus Pocock, 1894 es descrita para el estado Falcón, en el tramo occidental de la Cordillera de la Costa, Venezuela. Es la tercera especie del género registrada para el país, siendo el registro más al norte del género en el continente suramericano y el primero del orden para el estado Falcón.
\end{abstract}

Palabras clave: Thelyphonida, Thelyphonidae, Mastigoproctus, Suramérica, Neotrópico, Vinagrillo.

\section{ABSTRACT \\ A new species of Mastigoproctus Pocock, 1894 (Thelyphonida, Thelyphonidae) from northwestern Venezuela}

A new species of the genus Mastigoproctus Pocock, 1894 is described from Falcon state in northwestern Venezuela. This is the third Mastigoproctus species recorded for this country, the northernmost record of the genus in South America and the first record of the order for Falcon state.

Key words: Thelyphonida, Thelyphonidae, Mastigoproctus, South America, Neotropics, whip-scorpion.

\section{Introducción}

Posterior al trabajo de Weygoldt (1979), poco fue publicado en torno a la taxonomía de los vinagrillos de América del Sur (orden Thelyphonida) hasta comienzos de la década del 2000, cuando se evidenció un cambio en esta situación y varios autores comenzaron a producir contribuciones sobre fauna de vinagrillos suramericanos, centroamericanos y antillanos, describiendo géneros y especies nuevos, ampliando la distribución y/o aportando datos sobre especies poco conocidas

\footnotetext{
Sub Proyecto 1 / Red Antiveneno. Museo del Instituto de Zoología Agrícola, Facultad de Agronomía, Universidad Central de Venezuela, Apartado 2101-A, ZP 4579, Maracay, Edo. Aragua,Venezuela; osvaldovillarreal@gmail.com

2 Laboratório de Aracnologia, Museu Nacional, Universidade Federal do Rio de Janeiro,Quinta da Boa Vista s/n, São Cristóvão, Rio de Janeiro-RJ, Brasil. 20940-040.

$3 \quad$ Núcleo de Morfologia e Ultraestrutura de Vetores, Departamento de Entomologia, IOC / Fiocruz; agiupponi@gmail.com
} 
previamente (Armas, 2000, 2002; Armas \& Maes, 2000; Rowland, 2002; Rowland \& Adis, 2002; Ballesteros \& Francke, 2006; Víquez \& Armas, 2005, 2006a, 2006b, 2007; Armas et al., 2007; Giupponi \& Vasconcelos, 2008; Huff et al., 2008).

El género Mastigoproctus Pocock, 1894 es un grupo americano de vinagrillos con solo 10 especies descritas para América del Sur (Rowland, 2002; Viquez \& Armas, 2007). En Venezuela ha sido escasamente estudiado, con una especie registrada durante el siglo pasado y pobremente conocida: M. formidabilis Hirst, 1912, hasta que fue "redescubierta" en los Andes venezolanos y las estructuras genitales de ambos sexos fueron descritas (Armas et al., 2007). Poco tiempo después fue descrita una segunda especie para este país: M. ayalai Víquez \& Armas, 2007 proveniente del Escudo Guyanés (Víquez \& Armas, 2007).

En el presente trabajo se describe una nueva especie, conocida únicamente de una caverna en el noroccidente de Venezuela.

\section{Material y métodos}

Todas las medidas ofrecidas están en milímetros $(\mathrm{mm})$, el patrón de descripción fue adaptado de Weygoldt (1979), Armas (2002) y Víquez \& Armas (2005). Las ilustraciones, excepto la Fig. 17 (Armas et al., 2007) y la Fig. 18 (Víquez y Armas, 2007), fueron hechas utilizando una lupa estereoscópica con cámara de dibujo y procesadas con el programa informático Adobe Illustrator CS3. Las fotos fueron hechas con sendas cámaras digitales Nikon E5400 y Samsung Digimax A40, y procesadas usando el programa informático Adobe Photoshop CS3. La terminología empleada para la caverna es la de S.V.E. (2000).

El material tipo fue depositado en las siguientes instituciones: Museo de Historia Natural La Salle (MHNLS), Caracas, Venezuela; Museo del Instituto de Zoología Agrícola Francisco Fernández Yépez (MIZA), Maracay, Venezuela; y Museu Nacional (MNRJ), Universidade Federal do Rio de Janeiro, Rio de Janeiro, Brasil. Material no tipo fue examinado de las colecciones del Museu Nacional, Universidade Federal do Rio de Janeiro, Rio de Janeiro, Brasil, Museu de Zoologia, Universidade de São Paulo (MZSP) y Museo de Historia Natural La Salle, Caracas, Venezuela.

\section{Taxonomía}

Thelyphonidae Lucas, 1835

Mastigoproctinae Speijer, 1933

Mastigoproctus Pocock, 1894

Mastigoproctus abeli sp. nov.

(Figs. 1-16, 19, 20; Tabla 1)

Material tiPo: Holotipo macho: Venezuela: Falcón: Cerro la Misión, Cueva del Zumbador, 1051'26" Lat. N, 68³6'41" Long. W, 390 metros de altitud, 14-XI-1998, Villarreal, M. O., (MHNLS). Paratipos: hembra - Venezuela: Falcón: Cerro la Misión, Cueva del Zumbador, 1051'26" Lat. N, 68³6'41" Long. W, 390 metros de altitud, VII-2005, Villarreal, M.O. \& Carreño R. (MIZA 1286). Macho - Venezuela: Falcón: Cerro la Misión, Cueva del Zumbador, 1051'26" Lat. N, 68³6'41", Long. W, 390 metros de altitud, VII-2005, Villarreal, M.O. \& Carreño R. (MNRJ 8220).

Diagnosis: especie mediana con 37-44 $\mathrm{mm}$ de tamaño corporal (quelíceros-pigidio), que difiere de $M$. ayalai (omatoides irregulares y muy reducidos) por poseer los omatoides bien desarrollados y elípticos. Trocánteres de los pedipalpos con las espinas más distales ( $T d 4$ y $T d 5)$ sub iguales $(T d 4$ mucho mayor que $T d 5$ en $M$. alayai), posee pedipalpos con muchas estructuras en bajo relieve, en forma de pequeños cráteres (en $M$. ayalai son un poco más lisos) y el macho posee los pedipalpos mucho más cortos y robustos. Tibia con una espina ventrodistal corta de base ancha y puntiaguda (en M. ayalai esta espina es muy reducida y más roma). Difiere de $M$. formidabilis por poseer el gonópodo mucho más estrecho en relación al largo; los omatoides son semejantes, pero los machos de la especie nueva poseen los pedipalpos más cortos y robustos. Las hembras son claramente diferenciadas por la forma de los receptáculos seminales.

DESCRIPCIÓN DEL HOLOTIPO MACHO: Carapacho (Fig. 1) casi dos veces más largo que ancho, suavemente convexo, distancia entre el borde anterior y los ojos medios tres veces la distancia entre los ojos, con el montículo ocular muy bajo, rudimentario. Ojos laterales en posición casi vertical, próximos a los bordes laterales del carapacho, más distantes entre sí (las triadas) que en relación con los ojos medios. Toda la extensión del borde del carapacho posee un pliegue nítido, la porción frontal es redondeada con ápice romo, relativamente liso y con pocas cerdas, esa parte lisa cede lugar a una porción granulosa, compuesta de gránulos finos y gruesos distribuidos de forma aleatoria. Esa porción granulosa está separada de la parte lisa por una porción mediano-frontal en alto relieve con forma de "M", donde las "piernas de la M" están 
Tabla 1.- Medidas (en mm) de Mastigoproctus abeli sp. n. A, ancho; H, alto; L, longitud.

Table 1.- Measurements (in mm) of Mastigoproctus abeli $\mathrm{sp}$. n. A, width; $H$, height, $L$, length.

\begin{tabular}{|c|c|c|c|}
\hline & $\begin{array}{l}\text { Holotipo } \\
\text { MHNLS }\end{array}$ & $\begin{array}{c}\text { Paratipo } \\
\text { MIZA } \\
1286\end{array}$ & $\begin{array}{c}\text { Paratipo } \\
\text { MNRJ } \\
8220\end{array}$ \\
\hline L total (sin flagelo) & 44 & 37 & 42 \\
\hline Prosoma L/ A posterior & $17 / 9$ & $17 / 8$ & $16 / 7$ \\
\hline Pedipalpo, L & 23 & 28 & 23 \\
\hline Trocánter L/A & $6 / 6$ & $5 / 6$ & $5 / 6$ \\
\hline Fémur (L/A/H) & $8 / 4 / 4$ & $8 / 4 / 5$ & $6 / 4 / 4$ \\
\hline Patela (L/A/H) & $6 / 4 / 4$ & $6 / 5 / 5$ & $5 / 3 / 4$ \\
\hline Tibia (L/A/H) & $5 / 3 / 3$ & $5 / 3 / 4$ & $4 / 2 / 3$ \\
\hline Dedo movible (L) & 6 & 5 & 4,5 \\
\hline \multicolumn{4}{|l|}{ Patas } \\
\hline Trocánter I & 3 & 2 & 2 \\
\hline Fémur I & 11 & 11 & 9 \\
\hline Patela I & 15 & 15 & 10 \\
\hline Tibia I & 15 & 15 & 11 \\
\hline Tarso I & 11,5 & 11 & 8 \\
\hline Trocánter II & 3,5 & 1 & 1 \\
\hline Fémur II & 8,5 & 9 & 8 \\
\hline Patela II & 4,5 & 4,5 & 4 \\
\hline Tibia II & 8,5 & 7,5 & 7 \\
\hline Basitarso II & 1,5 & 1,5 & 1,5 \\
\hline Tarso II & 4,5 & 4 & 3,5 \\
\hline Trocánter III & 4 & 3 & 3 \\
\hline Fémur III & 8,5 & 8,5 & 8 \\
\hline Patela III & 4,5 & 4,5 & 4 \\
\hline Tibia III & 7,5 & 7 & 6,5 \\
\hline Basitarso III & 1,5 & 1,5 & 1,5 \\
\hline Tarso III & 4,5 & 4,1 & 3,5 \\
\hline Trocánter IV & 5 & 4,5 & 4 \\
\hline Fémur IV & 11 & 11 & 10,5 \\
\hline Patela IV & 5 & 5 & 4,5 \\
\hline Tibia IV & 11 & 10 & 10 \\
\hline Basitarso IV & 2 & 2 & 2 \\
\hline Tarso IV & 6 & 5 & 4,5 \\
\hline
\end{tabular}

ubicadas inmediatamente al lado (interno) de los ojos laterales, y su ápice termina exactamente detrás de los ojos medios. El borde posterior del carapacho es ligeramente cóncavo.

Esternón trisegmentado, con tritoesternón muy desarrollado y con forma de tridente estilizado, teniendo tres puntas anteriores romas, levemente curvadas, la central, se mete levemente entre las coxas del pedipalpo y las dos puntas laterales del esternón que terminan en el área entre las coxas pedipalpares y de la pata II. La porción posterior del tritosternón, se prolonga hasta el área central entre las coxas II y III, y termina en forma de gota. Mesoesternón muy reducido, pero visiblemente presente, de forma romboidal y con ápices redondeados, posicionado entre las coxas de las patas III y IV. Metaesternón de forma triangular con ápice romo que apunta hacia adelante inmediatamente detrás de las coxas IV.

Abdomen (Figs. 2, 3) oblongo, más largo que ancho, posee granulación fina y aspecto brillante, terguitos y esternitos con dos marcas paralelas en bajo relieve, que corresponden a las inserciones musculares. Pigidio más largo que ancho, de forma casi cilíndrica, con un par de omatoides pequeños y ovoides. Flagelo incompleto, con al menos 22 artículos verificables, largo y con abundantes cerdas largas y delgadas, de coloración rojiza (el del macho paratipo, también incompleto, posee 35 artículos). Órganos del flagelo (whip-organs) a partir del séptimo artículo, blanquecinos, de forma elipsoidal.

Pedipalpo (Figs. 4-7). Coxa muy desarrollada y observable solamente en vista ventral, plana, lisa y brillante, mucho más larga que ancha, de forma trapezoidal, con la base menor en contacto con el esternón y la base mayor formando la cámara preoral, armada en la base mayor con una apófisis grande que apunta para adelante (apófisis coxal) de base ancha, afinándose abruptamente, con la punta muy aguda y recurva hacia la cara interna, con muchas cerdas. Trocánter tan largo como ancho, dorsalmente con cinco espinas en la parte interna, siendo la proximal $(T d l)$ ligeramente menor que las demás, que son subiguales, la espina $T d 4$ es roma, y $T d 5$ dirigida anteriormente. Ventralmente con dos pequeñas espinas, siendo la más proximal $(T v 1)$ un poco menor que la más distal $(T \vee 2)$. El trocánter posee un área interna bien desarrollada, con una superficie plana con algunos gránulos y cerdas. Fémur corto, más largo que ancho, cutícula lisa y brillante, posee dorsalmente una espina interna diminuta meso-distal $(F d 1)$, ventralmente posee una espina medial $(F v l)$ notablemente mayor que $F d 1$. Entre las espinas dorsal y ventral existe una quilla de pequeños gránulos. Patela casi tan larga como ancha, de aspecto liso y brillante, con una gran apófisis espiniforme (apófisis patelar), dentada en el margen externo. Apófisis patelar tan larga como la patela, teniendo la base estrecha y la punta aguda. Tibia corta y ancha, con aspecto plano y redondeado (discoidal), cutícula lisa y armada con una apófisis grande y larga (apófisis tibial), de base larga que se afina abruptamente en una punta muy 
1

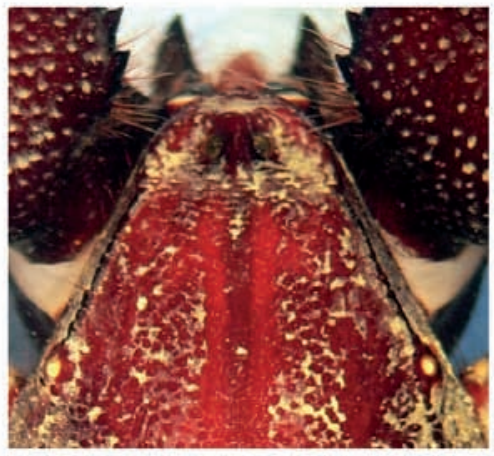

\section{2}

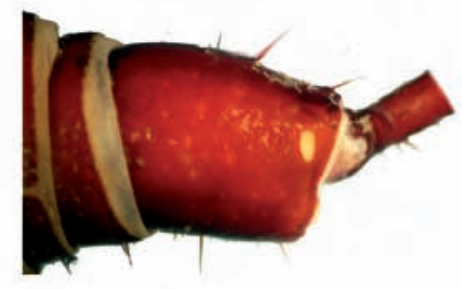

3

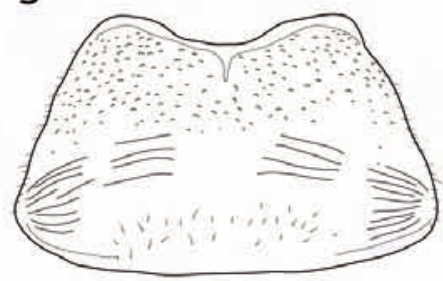

4

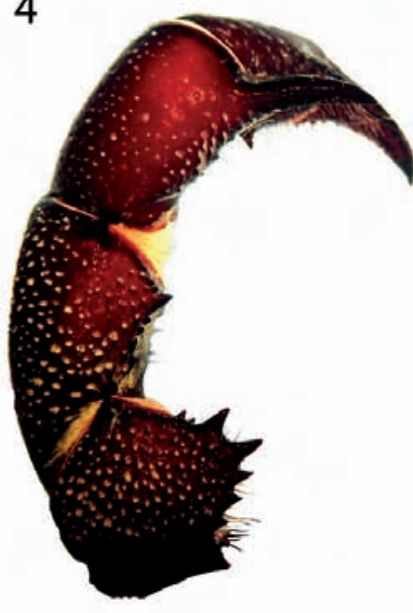

5
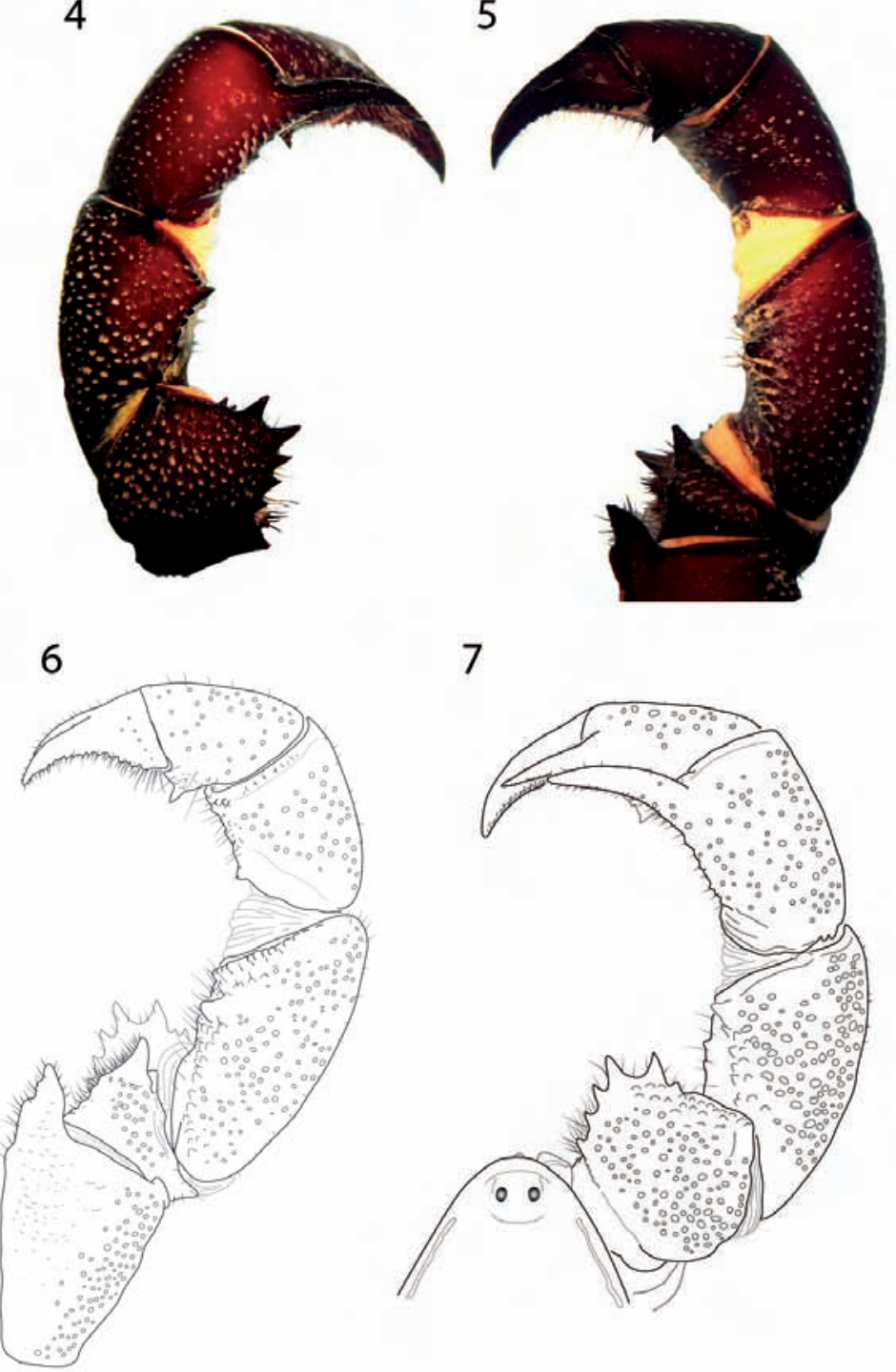

7

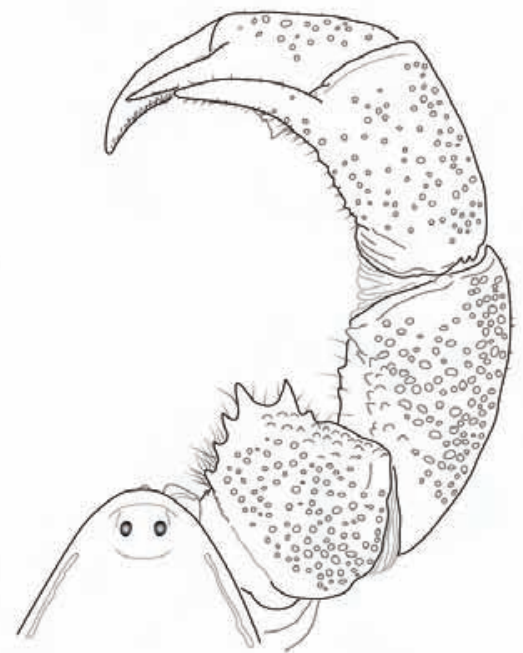

Figs. 1-7.- Mastigoproctus abeli sp. nov. Holotipo macho: 1) Carapacho. 2) Pigidio en vista lateral. 3) Esternito I, en vista ventral. 4-7) Pedipalpo, en vista dorsal y ventral.

Figs. 1-7.- Mastigoproctus abeli sp. nov. Male holotype: 1) Carapace. 2) Pygidium in lateral view. 3) Sternite I, in ventral view. 4-7) Pedipalp in dorsal and ventral view.

aguda. Apófisis con muchas cerdas en la porción interna, su longitud es aproximadamente la mitad del tamaño de la apófisis patelar y forma con el basitarso una pseudoquela. Basitarso en forma de garra recurva para su porción interna y con margen dorso-interno con varios dentículos en una fila, también recubierta de cerdas, con la punta muy aguda y de un largo similar al de la tibia.
Patas. Pata I con basitarso subdividido en nueve artejos. Fémur con un grupo de tubérculos conspicuos ventrodistales distribuidos en dos filas, los distales mayores. Tibia de las patas II-IV con solo un tricobotrio dorsodistal, y tibias de las patas I (patas anteniformes) con dos tricobotrios dorsodistales paralelos. Trocánteres de las patas IV armados con fuertes tubérculos dirigidos posteriormente. 
8

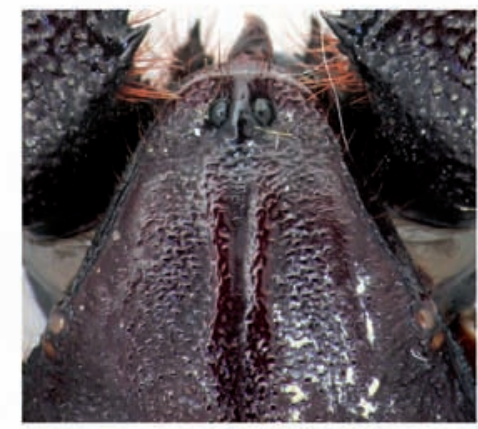

9

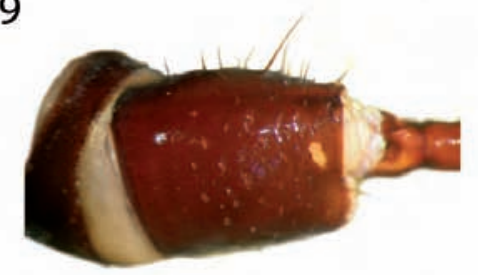

10

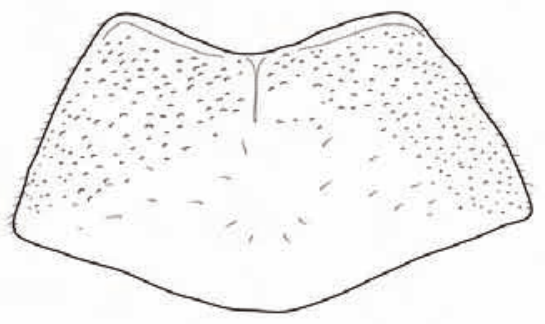

11

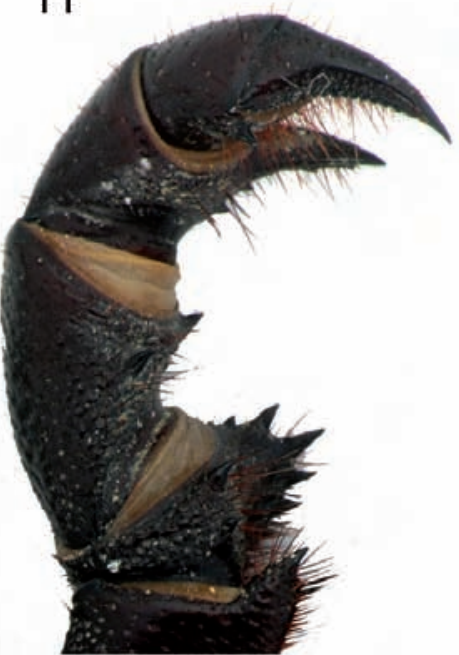

12
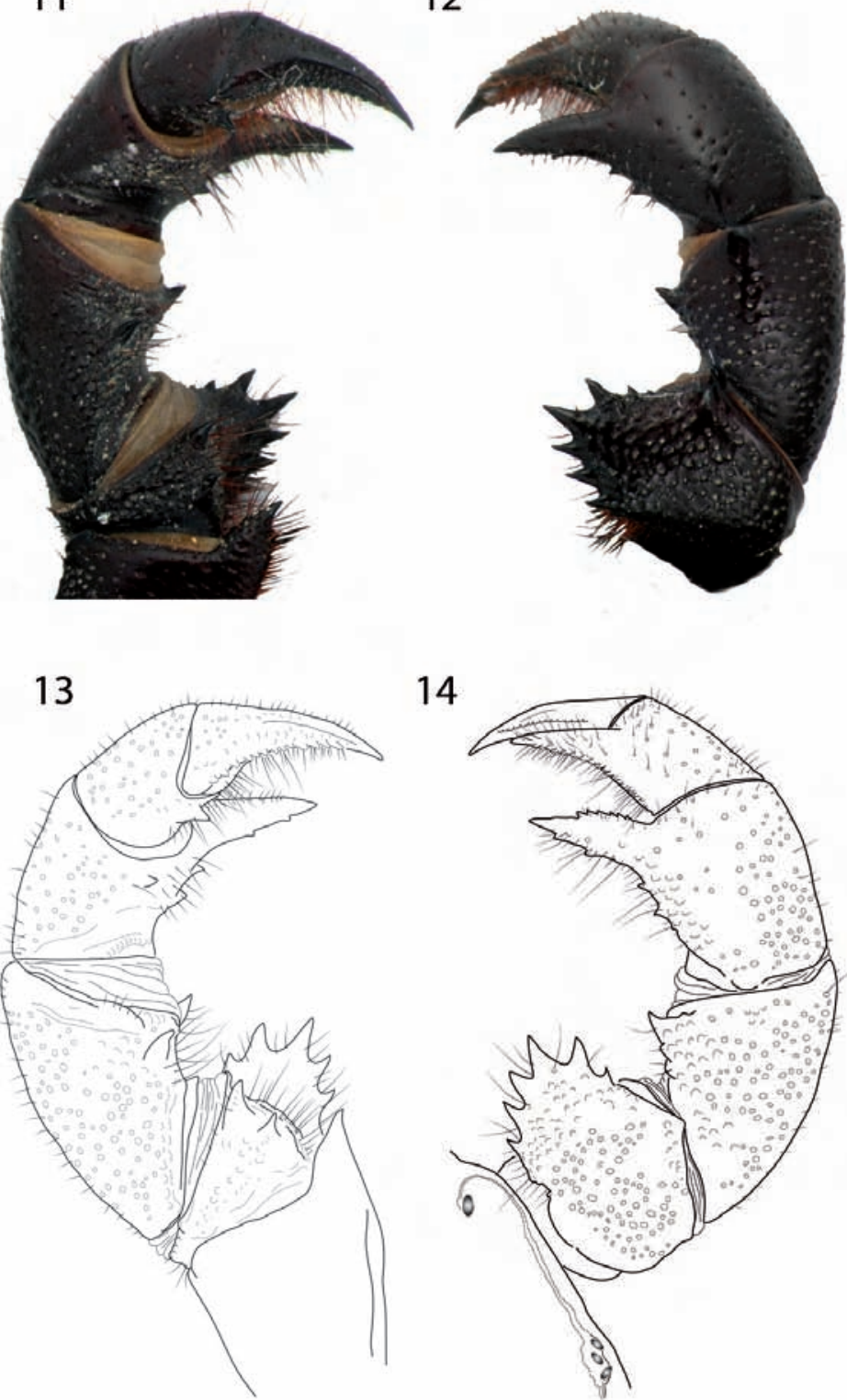

14

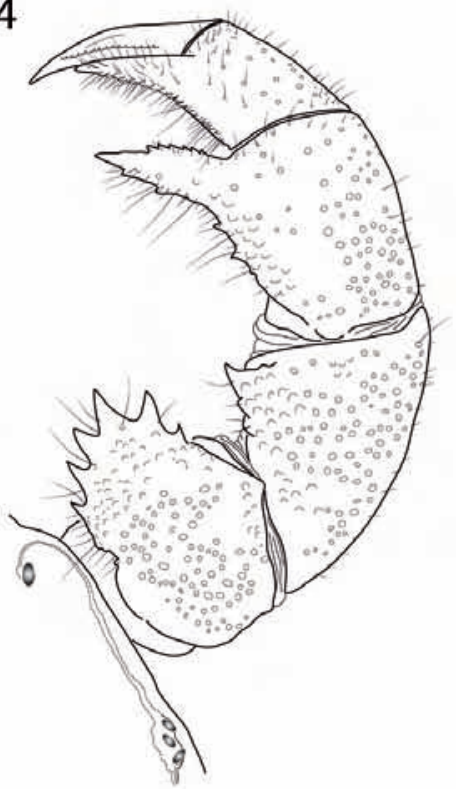

Figs. 8-14.- Mastigoproctus abeli sp. nov. Paratipo hembra: 8) Carapacho. 9) Pigídio en vista lateral. 10) Esternito I, en vista ventral. 11-14) Pedipalpo, en vista dorsal y ventral.

Figs. 8-14.- Mastigoproctus abeli sp. nov. Female paratype: 8) Carapace. 9) Pygidium in lateral view. 10) Sternite I, in ventral view. 11-14) Pedipalp in dorsal and ventral view.

Tibia de las patas III y IV ventralmente armadas con un espolón. Basitarso de las patas II- IV ventralmente armados con dos espolones.

Genitales (Fig. 15). Gonópodo simple, más ancho que largo, formado por un borde de cutícula espesa y oscura, casi negro, en forma de cuchara. Borde ante- rior irregular, procurvo y angulado; borde posterior cóncavo y regular. Parte interna casi recta, levemente cóncava; parte externa inclinada ligeramente cóncava. Al centro de estas paredes quitinizadas una región membranosa, aparentemente inflable, más alta hacia la parte medial, donde es levemente quitinizada. 

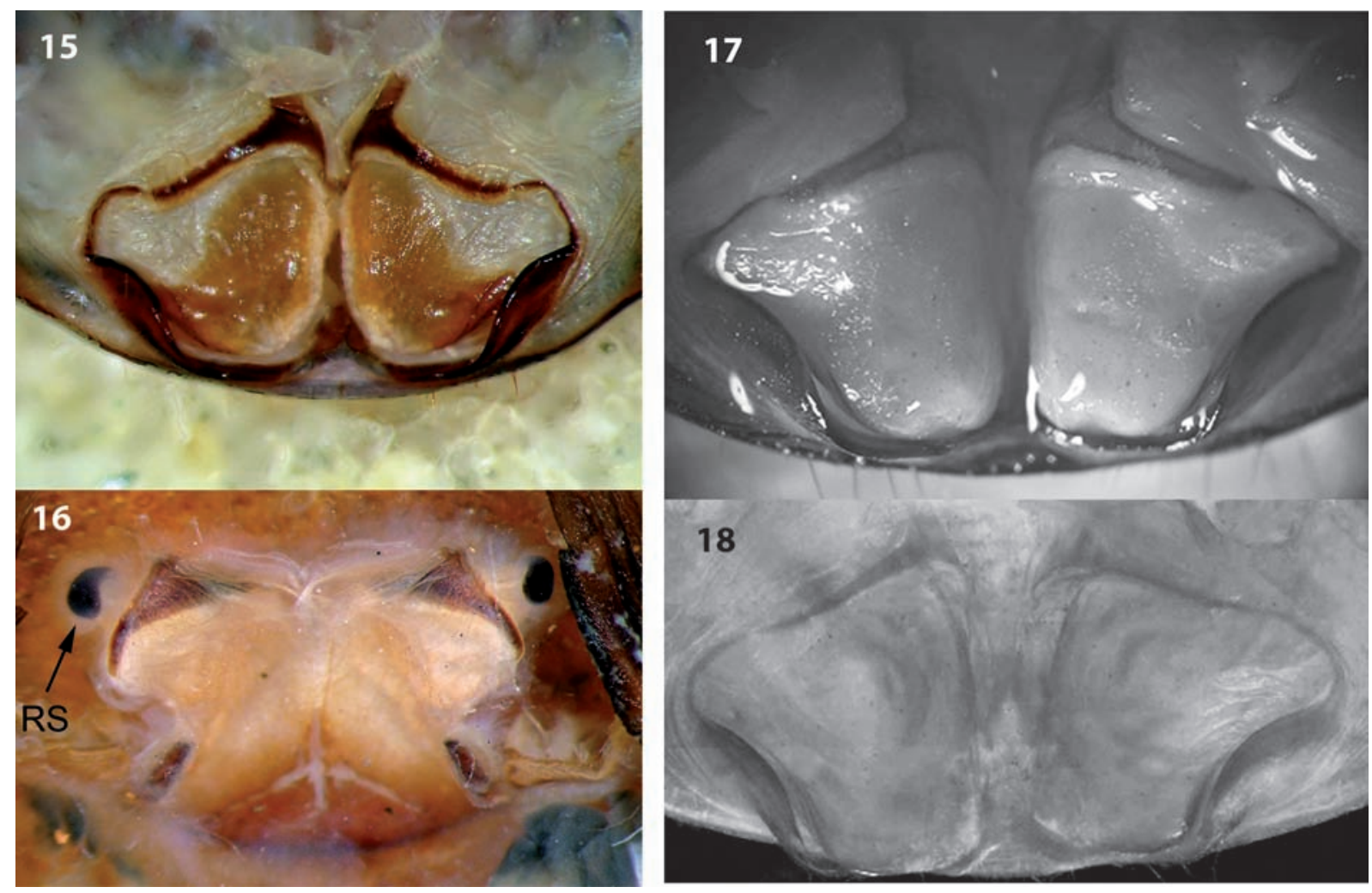

Figs. 15-18. - Mastigoproctus abeli sp. nov.: gonópodo del macho (15) y receptáculo seminales de la hembra (16). Gonópodos masculinos de 17) M. formidabilis (Venezuela, estado Trujillo, modificada de Armas et al., 2008) y de 18) M. ayalai (holotipo macho, tomada de Víquez \& Armas, 2007). Rs: receptáculo seminal.

Figs. 15-18. - Mastigoproctus abeli sp. nov.: Gonopods of the male (15) and seminal receptacles of the female (16). Gonopods of the males: 17) M. formidabilis (Venezuela, Trujillo, amended from Armas et al., 2008) and the 18) M. ayalai (male holotype, taken from Víquez \& Armas, 2007). Rs: seminal receptacle.

DESCRIPCIÓN DEL PARATIPO HEMBRA (Figs. 8-13, 16): Difiere del macho por poseer pedipalpos más cortos y robustos (Figs. 11-14); el trócanter menor, con Td6 grande y Tvl más larga y aguda; fémur más corto y ancho, con $F d l$ y $F v 1$ mayores y puntiagudas; patela más ancha, con la apófisis patelar más corta y ensanchada en la base, con sus márgenes laterales aserrados, con una pequeña espina ventrointerna; tibia con una fila de dentículos dorsales más conspicuos. El esternito I posee el borde posterior fuertemente procurvo, sin las estrías transversales notorias en la mitad posterolaeral. Receptáculos seminales con curvatura fuerte en la porción final, terminan en una punta roma oscura e inflada, en forma de esfera irregular, con el pliegue retrolateral casi recto (Fig. 16).

Coloración. Cefalotórax dorsalmente pardo rojizo oscuro, ventralmente un poco más claro.
Abdomen dorsalmente rojizo, un poco más claro que el cefalotórax, y ventralmente más fuertemente rojizo con bordes oscurecidos. Pedipalpos pardo rojizo muy oscuros, casi color vino tinto, con extremidades muy oscurecidas casi negras. Patas pardo rojizas brillantes.

ETIMOLOGía: La especie es dedicada al aracnólogo Abel Pérez González, especialista en sistemática de opiliones Samoideos, además organizador del Proyecto "Expedición Aracnológica Venezuela 2002" que en parte hizo posible este trabajo.

ECOTOPO E HISTORIA NATURAL: La Cueva del Zumbador (Fa.116 según el Catastro Espeleológico Nacional de Venezuela) (Fig. 19) se localiza en la zona de Yumare, a 15,5 km al SSW de Yaracal, en el Cerro La Misión, estado Falcón, se desarrolla en calizas de la Formación Capadare, a las cuales se les 

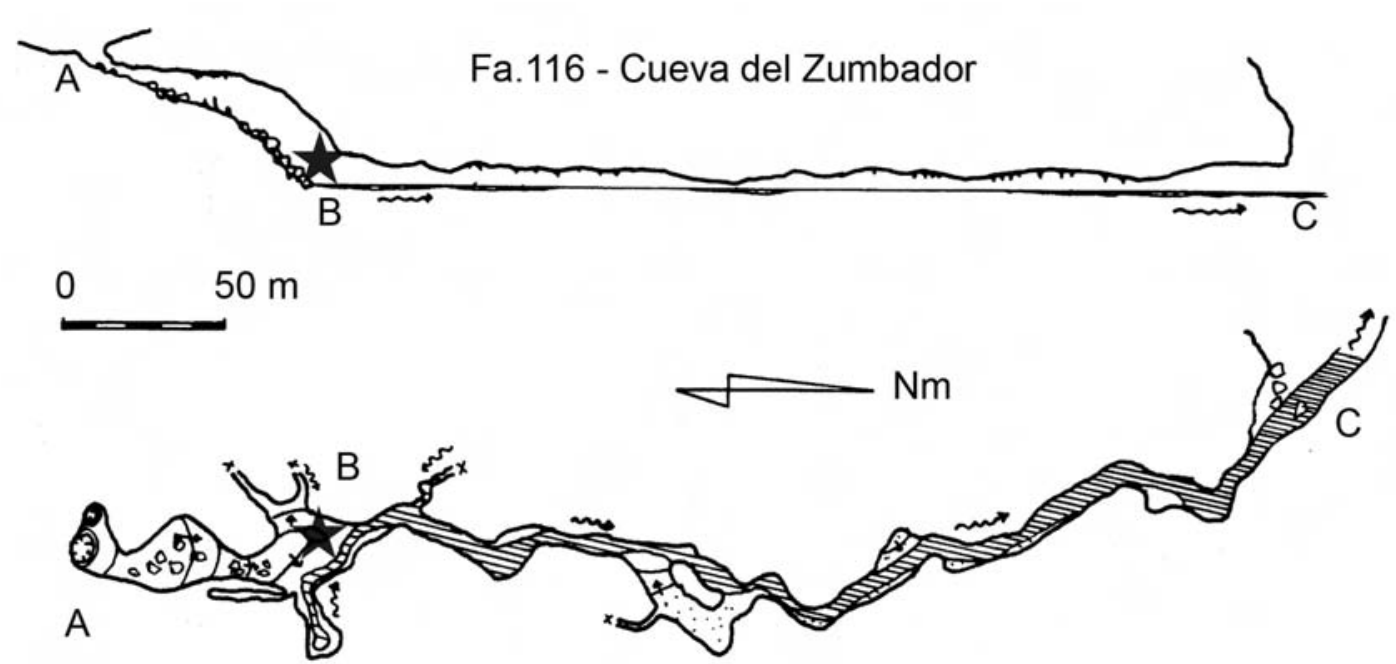

Fig. 19. - Mapa de la Cueva del Zumbador, en el que se indican los puntos de colecta. Modificado de SVE (2006).

Fig. 19.- Map of Cueva del Zumbador, which identifies the collection points. Modified from SVE (2006).

asigna una edad Mioceno Medio (Díaz de Gamero, 1985). Posee una extensión de 644 metros y un desnivel de 42 metros $(+42,-0)$. Altitud 390 metros. Esta es una caverna de origen calcáreo, hidrológicamente activa y tiene una gran riqueza de artrópodos vivientes y fósiles de diversos grupos de vertebrados del Pleistoceno, registrándose mamíferos fósiles como: Eremotherium laurillardi, Smilodon populator y Mixotoxodon larensis (Rincón, 2003) y un ave de la familia Pelagornithidae (Rincon \& Stucchi, 2003).

Solo tres ejemplares de esta nueva especie fueron observados y recolectados (durante dos visitas distintas a dicha caverna), todos los ejemplares fueron capturados en el mismo sector de la cueva, en zona de oscuridad total, cerca de $40 \mathrm{~m}$ de una entrada en forma de sima (Fig. 19). Otros arácnidos presentes en la caverna son: Avima cf. falconensis (GonzálezSponga, 1987) (Opiliones: Agoristenidae) y Heterophrynus cheirachantus (Gervais, 1842) (Amblypygi: Phrynidae). Los ejemplares de M. abeli sp. nov. fueron recolectados entre las 20:00 y 22:00 horas, próximos al cuerpo de agua.

\section{Discusión}

Mastigoproctus abeli sp. nov. es conocida únicamente de la localidad tipo, sin embargo, no posee ningún troglomorfismo evidente. La caverna es el nacimiento de un pequeño río en un bosque húmedo tropical, en un área con afloramientos de roca caliza y, aun cuando ejemplares epigeos de esta especie no se han recolectado, es probable que también habite en el bosque y que la caverna esté siendo utilizada por esta especie como área de forrajeo. No obstante, ningún esfuerzo de recolecta ha sido llevado a cabo en las inmediaciones de la cueva con la finalidad de capturar este vinagrillo.

Hasta el presente $M$. abeli $\mathbf{s p . ~ n o v . ~ e s ~ c o n o c i d a ~}$ solo de la región noroiental del estado Falcón, inventarios preliminares sobre la fauna hipogea de las formaciones kársticas más próximas (la Sierra de San Luis, localizada $130 \mathrm{Km}$ al oeste-noreste y Cuevas de la Península de Paraguaná, a 195 km noroeste), no registran ningún ejemplar del orden Thelyphonida (Chapman, 1980; Peck, 1982).

Tanto la morfología externa como la de los gonópodos masculinos son semejantes entre $M$. abeli sp. nov., M. formidabilis y $M$. ayalai, difiriendo todas estas especies levemente entre si, lo que hace suponer la relativa cercanía evolutiva entre ellas. La relación de largo/ancho de los gonópodos masculinos fue evaluada con base en las descripciones originales y fue detectado un área de no solapamiento en dicha relación, obteniéndose: $M$. formidabilis $=2,0 ; M$. alayai $=2,21 ; M$. abeli $\mathbf{s p}$. nov. $=1,73$ sin embargo la escasas muestras existentes y el desconocimiento de la variabilidad de esta estructura no permiten un análisis estadístico, 


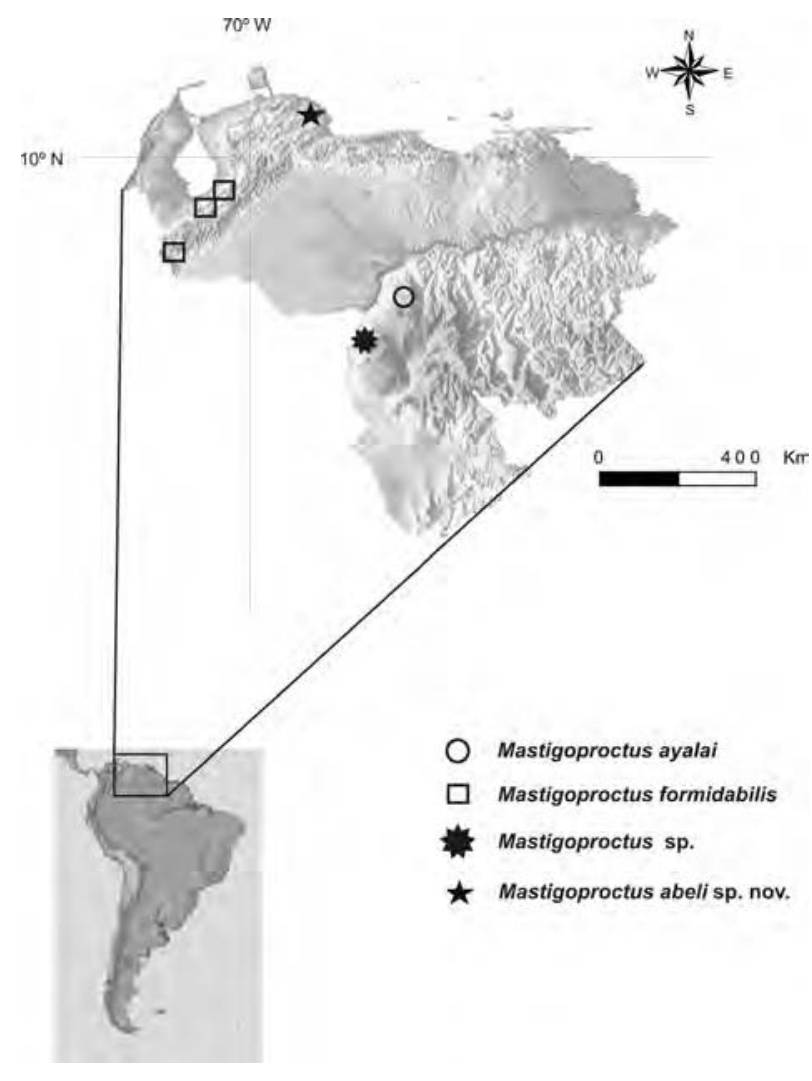

Fig. 20.- Mapa que muestra la distribución de Mastigoproctus en Venezuela.

Fig. 20.- Map showing the distribution of Mastigoproctus in Venezuela.

o una afirmación categórica, sin embargo pareciera que esta relación ancho largo podría ser usada en conjunto con otros caracteres como los receptáculos seminales o la morfología externa, en la taxonomía alfa del grupo.

Las hembras de la nueva especie, poseen un gonópodo claramente menos ancho en relación al largo que el observado en $M$. formidabilis y $M$. ayalai $(1,73 ; 2,0$ y 2,21 , respectivamente), adicionalmente, presentan el pliegue retrolateral casi recto, semejante a $M$. formidabilis, pero diferente al de $M$. ayalai el cual es cóncavo; los bordes laterales en su porción más distante del centro son casi paralelos en la nueva especie, convergentes y rectos en $M$. formidabilis y curvos y convexos en $M$. ayalai.

Con base en la morfología externa y genital, se puede hipotetizar una posible relación de cercanía evolutiva entre estas tres especies, sin embargo, un análisis sistemático general, incluyendo las restan- tes especies del género sería necesario para establecer un grado más preciso de parentesco entre ellas.

Con soporte en la propuesta de áreas biogeográficas de Morrone (2001) se puede decir que Mastigoproctus formidabilis habita en la Región Andina (Fig. 20), siendo el único representante del género que habita en esta bioregión; $M$. alayai habita en la región del Escudo de las Guayanas; $M$. abeli sp. nov. en la Sub-región caribeña y $M$. colombianus es conocida de la Sub-región Amazónica con distribución limitada por los Andes al oeste y por el Rio Orinoco al este (Provincia biogeográfica de Napo).

Mastigoproctus formidabilis aparentemente es un endemismo de los Andes venezolanos (Provincia biogeográfica de Páramo Norandino), sin embargo siempre ha sido recolectado a escasa altitud, asociada a los bosques del piedemonte. M. alayai está restringida a la Provincia biogeográfica de Imerí, limitada al norte por el Rio Orinoco, y al sur por el inicio de formaciones boscosas más cerradas $\mathrm{y}$ húmedas y por la Serranía de la Cerbatana.

Mastigoproctus abeli sp. nov. es conocida de los bosques de la porción oeste de la Cordillera de la Costa, en el noroeste de Venezuela. Estudios ecológicos serían necesarios para conocer el estado de las poblaciones de dicha especie.

\section{AGRADECIMIENTOS}

A Luis F. de Armas (La Habana) y Carlos Víquez (INBio, Costa Rica) por sus valiosos comentarios y sugerencias sobre el manuscrito. Al CNPq por la beca de doctorado concedida (A.P.L.G.). A Eduardo Gomes de Vasconcelos (MNRJ, Brasil) por las críticas y sugerencias sobre una versión temprana del manuscrito. A Quintín Arias (MIZA, Venezuela) por las facilidades prestadas durante la realización de las figuras $7-10$. A Rafael Carreño (SVE) por la colaboración en campo. A Celsa Señaris y Daniel Lew (MHNLS, Venezuela) por las facilidades para la revisión del material depositado en el MHNLS.

\section{Referencias}

ARMAS, L.F. DE, 2000 Los vinagrillos de Cuba (Arachnida: Uropygi: Thelyphonidae). Poeyana, 469:1-10.

ARMAS, L.F. DE, 2002. Nueva especie de Thelyphonellus (Thelyphonida: Thelyphonidae) de La Española, Antillas Mayores. Revista Ibérica de Aracnología, 5: $39-42$.

ARMAS, L.F. DE \& MAES, J.-M., 2000. Uropygi, un orden de Arachnida (Chelicerata) nuevo para Nicaragua. Revista Nicaragüense de Entomología, 50:13-15.

Armas, L.F. DE, Villarreal Manzanilla, O. \& Víquez, C., 2007. Redescubrimiento de Mastigoproctus formidabilis Hirst, 1921 (Thelyphonida: Thelyphonidae) 
en Venezuela. Boletín de la Sociedad Entomológica Aragonesa, 40: 423-425.

Ballesteros, A. \& Francke, O. F., 2006. Mastigoproctus lacandonensis, especie nueva de vinagrillo (Thelyphonida, Arachnida) de la Selva Lacandona, Chiapas, México. Entomología Mexicana, 5: 156-161.

CHAPMAN, P., 1980. The invertebrate fauna of caves of the Serranía de San Luis, Edo, Falcón, Venezuela. Transaction Caves Research Association, 7(4): 179199.

DíAz DE GAMERO, M. L., 1985. Estratigrafía de Falcón Nororiental. VI Congreso Geológico de Venezuela. (Caracas - Venezuela, 1985), Memoria I: 454-502.

Giupponi, A. P. L. \& VAsconcelos, E. G., 2008. Uma nova espécie do gênero Thelyphonellus Pocock, 1894 para a Colômbia (Arachnida: Thelyphonida: Thelyphonidae). Revista Ibérica de Aracnología, 16: 17-22.

Huff, J. C., Víquez, C. \& Prendini, L., 2008. Redescription of Mimoscorpius pugnator (Butler, 1872) (Arachnida: Thelyphonida), with first description of the female. American Museum Novitates, 3633: 1-9.

Mello-Leitão, C. F. DE, 1931. Pedipalpos do Brasil e algumas notas sobre a ordem. Arquivos do Museu Nacional, Rio de Janeiro, 33: 7-72.

Morrone, J. J., 2001. Biogeografía de América Latina y el Caribe. Manuales \& Tesis SEA, 3: 1-147.

PECK, S. B., 1982. A contribution to the knowledge of the invertebrate cave faunas of Venezuela: Invertebrate faunas of tropical American caves, part. 4. International Journal of Speleology, 12: 75-81.

RinCón, R. A. D., 2003. Los mamíferos fósiles del pleistoceno de la Cueva del Zumbador (Fa.116), Estado Falcón, Venezuela. Boletín de la Sociedad Venezolana de Espeleología, 37: 18-26.

Rincón, R.A.D. \& STuCChi, M., 2003. Primer registro de la familia Pelagornithidae (Aves: Pelicaniformes) para Venezuela. Boletín de la Sociedad Venezolana de Espeleología, 37: 27-30.

Rowland, J. M., 2002. Review of the South American whip scorpions (Thelyphonida: Arachnida). Amazoniana, 17(1/2):187-204.

Rowland, J. M. \& ADIS, J., 2002. 4.11. Uropigy (Thelyphonida). In: J. Adis (ed). Amazonian Arachnida and Myriapoda. Pensoft. Sofia \& Moscow: 449-456.

Rowland, J. M. \& CoOKE, J. A. L., 1973. Systematic of the arachnid order Uropygida (= Thelyphonida). Journal of Arachnology, 1: 55-71.

S.V.E. (Sociedad Venezolana de Espeleología), 2000. Catastro Espeleológico Nacional. Boletín de la Sociedad Venezolana de Espeleología, 34: 30.

Víquez, C. \& Armas, L. F. DE, 2005. Dos nuevos géneros de vinagrillos de América Central y las Antillas (Arachnida: Thelyphonida). Boletín de la Sociedad Entomológica Aragonesa, 37: 95-98.
Víquez, C. \& Armas, L. F. DE, 2006a. Un nuevo género y dos nuevas especies de vinagrillos centroamericanos (Arachnida: Thelyphonida). Boletín de la Sociedad Entomológica Aragonesa, 38: 37-41.

Víquez, C. \& ARMAS, L. F. DE, 2006b. Los vinagrones de Guatemala (Arachnida: Thelyphonida). In: E. B. Cano (ed.). Biodiversidad de Guatemala. Vol. 1. Universidad del Valle de Guatemala. Guatemala: 299-306.

Víquez, C. \& Armas, L. F. DE, 2007. A new species of Mastigoproctus (Thelyphonida: Thelyphonidae) from Venezuela. Zootaxa, 1463: 39-45.

Weygoldt, P., 1979. Thelyphonellus ruschii n. sp. und die taxonomsiche Stellung von Thelyphonellus Pocock, 1979. Senckenbergiana Biologica, 60(1/2): 109-114.
Recibido, 10-VII-2009 Aceptado, 23-XI-2009 Publicado, 31-XII-2009 